\title{
Adiponectin suppresses endoplasmic reticulum stress in nonalcoholic steatohepatitis
}

\author{
TAKATO UENO $^{1}$, ANNA NAKAMURA ${ }^{1}$, HITOMI NAKAYAMA ${ }^{2}$, SHUICHI OTABE $^{2}$, XIAOHONG YUAN $^{2}$, \\ TOMOKA FUKUTANI ${ }^{2}$, HIDEKI IWAMOTO ${ }^{3}$, TORU NAKAMURA ${ }^{3}$, HIRONORI KOGA $^{3}$, \\ TAKUJI TORIMURA ${ }^{3}$, MICHIO SATA ${ }^{3}$ and KENTARO YAMADA ${ }^{2}$ \\ ${ }^{1}$ Research Center for Innovative Cancer Therapy, Kurume University; ${ }^{2}$ Division of Endocrinology and Metabolism, and \\ ${ }^{3}$ Division of Gastroenterology, Department of Medicine, Kurume University School of Medicine, Kurume, Fukuoka, Japan
}

Received July 24, 2011; Accepted August 22, 2011

DOI: $10.3892 / \mathrm{etm} .2011 .348$

\begin{abstract}
In this study, we examined whether adiponectin suppresses endoplasmic reticulum (ER) stress in nonalcoholic steatohepatitis (NASH) using male transgenic mice expressing nSREBP-1c in adipose tissue, nSREBP-1c/adiponectin doubletransgenic mice expressing human adiponectin in the liver, and wild-type male mice as the control. Histological findings similar to those observed in liver specimens from patients with NASH were observed in the livers from the nSREBP-1c transgenic mice at 30 weeks of age. By contrast, the NASH-like liver histology was markedly attenuated in age-matched nSREBP-1c/adiponectin double-transgenic mice. The nSREBP-1c/adiponectin double-transgenic mice showed human adiponectin production in the liver and a restored circulating human adiponectin level. Human adiponectin messenger ribonucleic acid (mRNA) expression in the liver was identified in the nSREBP-1c/adiponectin double-transgenic mice, but adiponectin receptor 1 and 2 mRNA expression in the liver was normal. TNF $\alpha$ mRNA was decreased in the liver of the nSREBP-1c/adiponectin doubletransgenic mice compared with the nSREBP-1c transgenic mice. The protein expressions of X-box-binding protein-1, activating transcription factor 4, acetyl-CoA carboxylase, $\mathrm{TNF} \alpha$ and $\mathrm{NF} \kappa \mathrm{B}$ were down-regulated in liver tissues from the nSREBP-1c/adiponectin double-transgenic mice. Mouse adiponectin and activating transcription factor 6 expressions were almost the same in the three groups. Post-load plasma glucose levels were significantly lower in the nSREBP-1c/adiponectin double-transgenic mice compared with the nSREBP-1c transgenic mice. These results indicate that adiponectin expressed in the liver suppresses ER stress and attenuates hepatic steatosis, inflammation and insulin resistance in NASH. Adiponectin may open the way to novel therapies for human NASH.
\end{abstract}

Correspondence to: Dr Takato Ueno, Research Center for Innovative Cancer Therapy, Kurume University, 67 Asahi-machi, Kurume, Fukuoka 830-0011, Japan

E-mail: takato@med.kurume-u.ac.jp

Key words: nonalcoholic steatohepatitis, adiponectin, endoplasmic reticulum stress

\section{Introduction}

The liver plays a central role in whole-body lipid metabolism by governing the synthesis, oxidization, transport and excretion of lipids. Unfolded protein response (UPR) was identified as a signal transduction system that is activated by endoplasmic reticulum (ER) stress (1). ER stress and activation of UPR have been linked to numerous human disorders, including obesity, type 2 diabetes and cancer (2). In addition, Rutkowski et al showed that unresolved ER stress contributes to metabolic dysfunction and hepatic steatosis (3).

UPR is a signaling system emanating from ER that is activated when ER protein folding is disturbed (4). Previous studies have revealed novel diverse functions of mammalian UPR, including its role in hepatic lipid metabolism (3). UPR activation has been observed in fatty liver diseases, suggesting the induction of ER stress in these conditions (5). Previous studies have demonstrated that ER stress activates the sterol regulatory element-binding proteins (SREBPs), transcription factors involved in de novo lipid biosynthesis (6). SREBPs play a significant role in cholesterol metabolism and LDL receptor expression (SREBP-2), as well as fatty acid and triglyceride biosynthesis (SREBP-1) (7). We have previously reported that transgenic mice expressing nuclear SREBP-1c (nSREBP-1c) in adipose tissue under the control of the aP2 promoter, an inherited lipodystrophic model with insulin resistance and fatty acids, spontaneously develop steatohepatitis (8).

Adiponectin is a hormone mainly produced by adipose tissue (9). Experimental studies have suggested that adiponectin plays a major role in the pathophysiology of insulin resistance and metabolic lipid storage and lipolysis in insulin-sensitive tissues, which may induce an increased flux of free fatty acids from adipose tissue to the liver and cause steatosis (10). Adiponectin plays a major role in the pathophysiology of insulin resistance and metabolic syndrome.

We have previously reported that the nSREBP-1c/adiponectin double-transgenic mice show hepatic adiponectin transgenically expressed in the liver, and nonalcoholic steatohepatitis (NASH)-like hepatic lesions were markedly attenuated in age-matched double-transgenic mice (11). In addition, Awazawa et al showed that adiponectin suppresses hepatic SREBP1c expression in an adiponectin receptor 
(AdipoR)1/LKB1/AMPK-dependent pathway (12). In this study, we examined whether adiponectin suppresses ER stress in NASH.

\section{Materials and methods}

Preparation of nSREBP-1c transgenic mice and nSREBP-1c/ adiponectin double-transgenic mice. Transgenic mice (C57BL/6 background) expressing nSREBP-1c in adipose tissue (13) were purchased from Jackson Laboratory (Bar Harbor, ME, USA), and bred in our laboratory by crossing with wild-type C57BL/6 mice (Nippon Clea, Shizuoka, Japan). Generation of transgenic mice expressing full-length human adiponectin in the liver of C57BL/6 background mice was previously described (14). Female mice that expressed nSREBP-1c in adipose tissue were crossed with adiponectin-expressing male mice to produce a nSREBP-1c/adiponectin double-transgenic line. The doubletransgenic mice were identified by polymerase chain reaction (PCR) of tail DNA using nSREBP-1c-specific primers (5'-CTA CATTCGCTTTCTGCAAC-3' and 5'-ATAGAAGGACACC TAGTCAG-3') and human adiponectin transgenic specific primers (5'-TGAATTCGGGCTCAGGATGCTGTTGCT-3' and 5'-AGGATCCTGATCAGTTGGTGTCATGGTA-3'). Male mice heterozygous for nSREBP-1c and human adiponectin were used in the following experiments. All mice were fed standard mouse chow $(347 \mathrm{kcal} / 100 \mathrm{~g}$, protein $24.9 \mathrm{~g} / 100 \mathrm{~g}$, fat $4.6 \mathrm{~g} / 100 \mathrm{~g}$; Nippon Clea) and water ad libitum. Body weight was measured prior to sacrifice. All procedures were approved by the Ethics Review Committee for Animal Experimentation of Kurume University School of Medicine.

Biochemical assays. Aspartate aminotransferase (AST), alanine aminotransferase (ALT), triglyceride and total cholesterol levels were determined by spectrophotometric enzyme assays using peroxidase, lipoprotein lipase and cholesterol oxidase, respectively (Wako Ltd., Osaka, Japan). Glucose tolerance was assessed using an intraperitoneal glucose tolerance test (IPGTT). The IPGTT was performed by injecting glucose $(1 \mathrm{~g} / \mathrm{kg}$ in $10 \%$ solution) intraperitoneally in overnight-fasted mice. Glucose levels in blood obtained from the tail veins were measured by glucose dehydrogenase methods using Free Style (Nipro, Osaka, Japan) at 0, 30, 60 and 120 min following glucose injection. Serum levels of mouse leptin and adiponectin were measured with enzyme-linked immunosorbent assay kits from R\&D (Oxon, United Kingdom) and AdipoGen (Seoul, Korea), respectively.

Light microscopy. Paraffin-embedded sections of the liver were stained with hematoxylin and eosin for standard microscopy or the Azan-Mallory stain to observe the location of the extracellular matrix in the liver tissues. The specimens were reviewed by a hepatopathologist. Each specimen was assigned to one of the following histological subgroups for the purposes of comparative analysis: type 1, fatty liver alone, which was predominantly macrovesicular in more than $33 \%$ of the lobules; type 2, fat accumulation and lobular inflammation; type 3 , fat accumulation and ballooning hepatocytes; type 4 , fat accumulation, ballooning hepatocytes, and either Mallory's hyaline or fibrosis. We dealt with types 3 and 4 as NASH, as previously described by Matteoni et al (5).
Electron microscopy. Passaged and cryopreserved HSCs were fixed in $1 \%$ glutaraldehyde for $1 \mathrm{~h}$ at $4{ }^{\circ} \mathrm{C}$, and post-fixed in $1 \%$ osmic acid for $1 \mathrm{~h}$ at $4^{\circ} \mathrm{C}$, dehydrated with ethanol and embedded. They were then sectioned and stained with lead citrate. The samples were then observed under an electron microscope (H-7650, Hitachi, Tokyo, Japan).

Real-time PCR analysis. For real-time quantitative PCR, the messenger ribonucleic acid (mRNA) levels of mouse adiponectin and AdipoR1 and 2, and human adiponectin were assayed using the 7000 sequence detection system ABI Prism sequence detector (Applied Biosystems, Tokyo, Japan), and the double-strand specific dye SYBR-Green (Applied Biosystems). The PCR conditions and cycles were as follows: initial DNA denaturations for $10 \mathrm{~min}$ at $95^{\circ} \mathrm{C}$, followed by 40 cycles of denaturation at $95^{\circ} \mathrm{C}$ for $15 \mathrm{sec}$, followed by an annealing step and then extension at $60^{\circ} \mathrm{C}$ for $1 \mathrm{~min}$. Each point was performed in triplicate. To ensure that the primers produced a single and specific PCR amplification product, a dissociation curve was generated during the PCR cycle and only primers with a unique dissociation peak were selected, followed by migration on a $2 \%$ agarose gel to ensure that the PCR product was unique. The amplification efficiency for each primer pair was calculated. The expression level of each gene was adjusted by the level of GAPDH and expressed as the ratio to GAPDH.

Western blot analysis. To determine whether there was any synergy between human and mouse adiponectins, TNF receptor 1 (TNFR1), pNFkB, acetyl-CoA carboxylase (pACC) and ER stress-related agents, such as X-box-binding protein-1 (XBP-1) and activating transcription factor-4 (ATF-4) and ATF-6, we performed Western blot analysis of whole cell protein extracts. Cells were lysed in RIPA buffer.Equal amounts of protein were separated on $10 \%$ SDS-polyacrylamide gel, and then blotted on a PVDF membrane. The membrane was immunoblotted. Immunoreactive bands were visualized using ECL detection reagents. The cells were incubated in a RIPA lysis buffer for $20 \mathrm{~min}$. Following determination of protein concentrations using a BCA protein assay kit (Pierce, Rockford, IL, USA), the samples were separated by $10 \%$ sodium dodecyl sulphate-polyacrylamide gel electrophoresis, and electroblotted onto nitrocellulose membranes. First they were reacted with primary antibodies and then with peroxidase-conjugated secondary antibody (dilution 1:1000) (GE Healthcare, Buckinghamshire, UK). Antigens were visualized by enhanced chemiluminescence using the ECL Western blotting detection system (Amersham, San Francisco, CA, USA).

Statistical analysis. Numerical data were expressed as the means \pm SD. Unpaired Student's t-test was performed to assess statistical significance between groups. A value of $\mathrm{P}<0.05$ was considered to be statistically significant.

\section{Results}

Biochemical assays. At 20 weeks of age, serum AST and ALT levels were elevated in the nSREBP-1c transgenic mice compared with the wild-type and nSREBP-1c/adiponectin transgenic mice. AST levels were highest in the nSREBP-1c 
transgenic mice, and were significantly reduced in the nSREBP-1c/adiponectin transgenic mice. ALT elevation was attenuated to normal levels in the nSREBP-1c/adiponectin transgenic mice, although the difference was not statistically significant (Fig. 1A). No significant difference was observed in total cholesterol or triglyceride levels between the nSREBP-1c transgenic mice and the nSREBP-1c/adiponectin transgenic mice (Fig. 1B).

The intraperitoneal glucose $(1 \mathrm{~g} / \mathrm{kg}$ in $10 \%$ solution) tolerance test performed at 20 weeks of age showed significantly lower plasma glucose levels in the nSREBP-1c/adiponectin transgenic mice than in the nSREBP-1c transgenic mice at 30 and $60 \mathrm{~min}$ after glucose load (Fig. 2).

Circulating endogenous mouse adiponectin levels were slightly but significantly higher in the nSREBP-1c/adiponectin transgenic mice than in the nSREBP-1c transgenic mice (Fig. 3A). Human adiponectin levels in the nSREBP-1c/adiponectin transgenic mice at 20 weeks of age were significantly higher than in the other two groups (Fig. 3B).

Light microscopy. For light microscopy, sections were stained with hematoxylin and eosin staining or Mallory's Azan. Fat droplets, cell infiltration and pericellular fibrosis were decreased in the nSREBP-1c/adiponectin transgenic mice (Fig. 4A and B) compared with the nSREBP-1c transgenic mice (Fig. 4C and D).

Electron microscopy. The livers of SREBP-1c transgenic mice contain hepatocytes with degenerated nuclei, and small and large fat droplets. Enlarged ERs are visible in the magnified image (Fig. 5A). However, hepatocytes in the livers of the nSREBP-1c and adiponectin double-transgenic mice contain a few small fat droplets in the cytoplasm. However, the nucleus and organelles, such as mitochondria and ER, are similar to those of normal hepatocytes. These findings show that the ER stress was decreased in the double-transgenic mice (Fig. 5B).

Real-time PCR analysis. Real-time PCR analysis showed that human adiponectin expression was significantly increased in the liver of nSREBP-1c/adiponectin transgenic mice compared with the wild-type and nSREBP-1c transgenic mice (Fig. 6A). However, there was no significant difference in the mouse adiponectin mRNA expression between the wild-type, nSREBP-1c transgenic mice and nSREBP-1c/adiponectin transgenic mice (Fig. 6B).

We observed no significant difference between the nSREBP-1c transgenic mice and nSREBP-1c/adiponectin transgenic mice in mouse AdipoR1 and 2 expression levels (Fig. 6C and D).

Western blot analysis. In Western blot analysis, TNFR1 and phosphorylated $\mathrm{NF \kappa B}$ were decreased in the liver of the nSREBP-1c/adiponectin transgenic mice compared with the nSREBP-1c transgenic mice. It can be assumed that adiponectin suppresses the inflammation through the TNFR1 and $\mathrm{NF} \kappa \mathrm{B}$ pathway in the liver of the nSREBP-1c/adiponectin transgenic mice (Fig. 7A).

In addition, the protein expressions of XBP-1, ATF-4 and phosphorylated ACC were decreased in the liver of the nSREBP-1c/adiponectin transgenic mice compared with
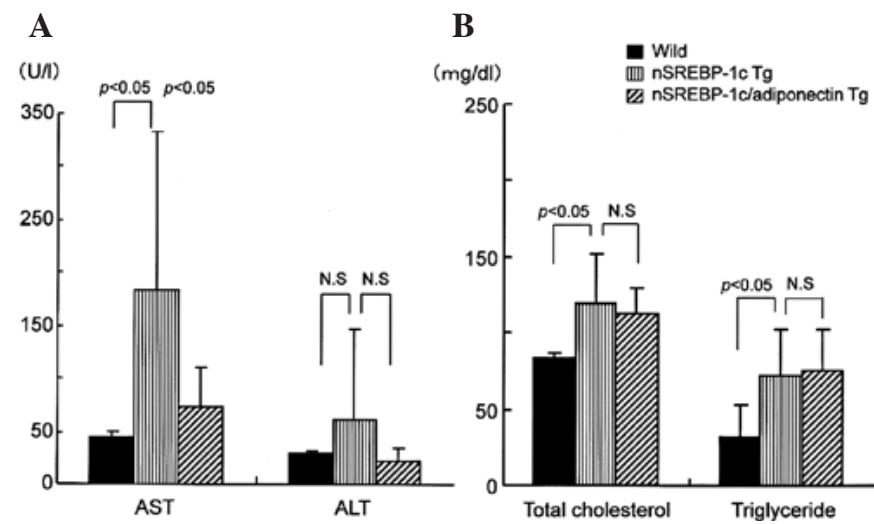

Figure 1. Serum transaminase and lipid levels at 20 weeks of age. (A) Serum AST and ALT levels were elevated in the nSREBP-1c transgenic mice compared with the wild-type and nSREBP-1c/adiponectin transgenic mice. AST levels were highest in the nSREBP-1c transgenic mice, and were significantly reduced in the nSREBP-1c/adiponectin transgenic mice. ALT level was normal in nSREBP-1c/adiponectin transgenic mice, although the difference compared with nSREBP-1c transgenic mice was not statistically significant. (B) No significant difference was observed in the total cholesterol or triglyceride levels between the nSREBP-1c transgenic mice and the nSREBP-1c/ adiponectin transgenic mice.

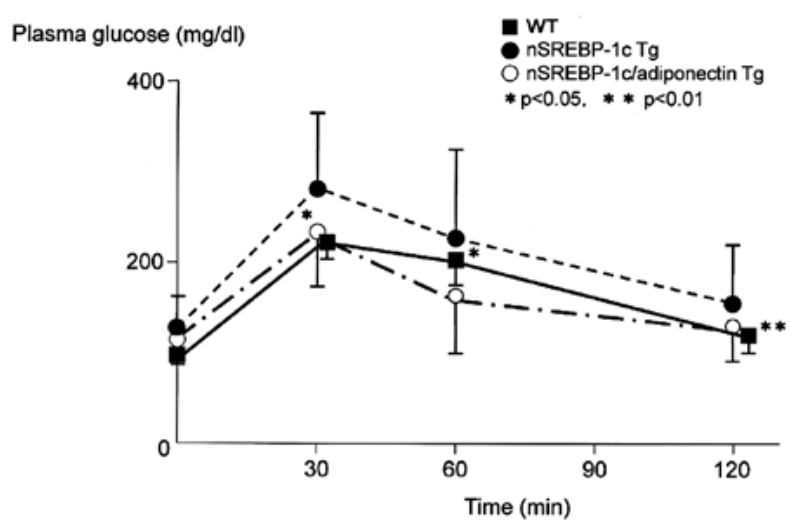

Figure 2. Intraperitoneal glucose tolerance test at 20 weeks of age. Intraperitoneal glucose ( $1 \mathrm{~g} / \mathrm{kg}$ in $10 \%$ solution) tolerance test performed at 20 weeks of age showed significantly lower plasma glucose levels in the nSREBP-1c/ adiponectin transgenic mice than in the nSREBP-1c transgenic mice at 30 and 60 min after glucose load.

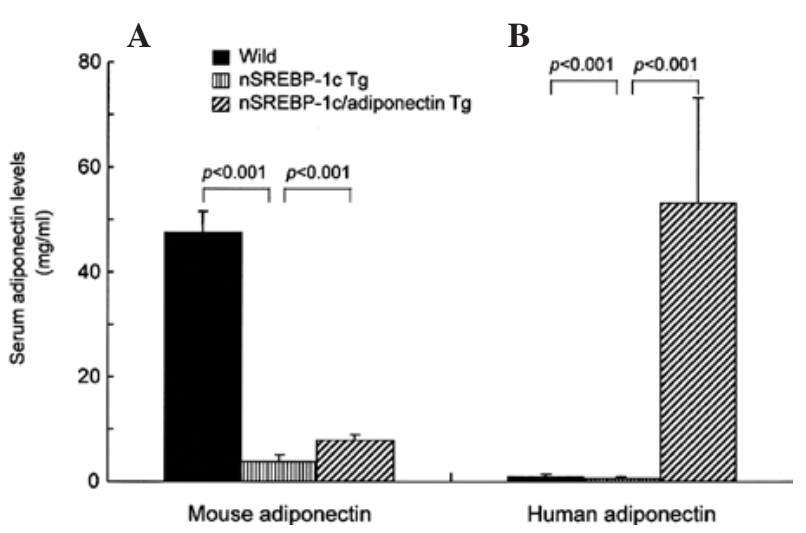

Figure 3. Circulating endogenous mouse adiponectin levels at 20 weeks of age. (A) Circulating endogenous mouse adiponectin levels were slightly but significantly higher in the nSREBP-1c/adiponectin transgenic mice than in the nSREBP-1c transgenic mice. (B) Human adiponectin levels of the nSREBP1c/adiponectin transgenic mice at 20 weeks of age were significantly higher than in the other two groups. 

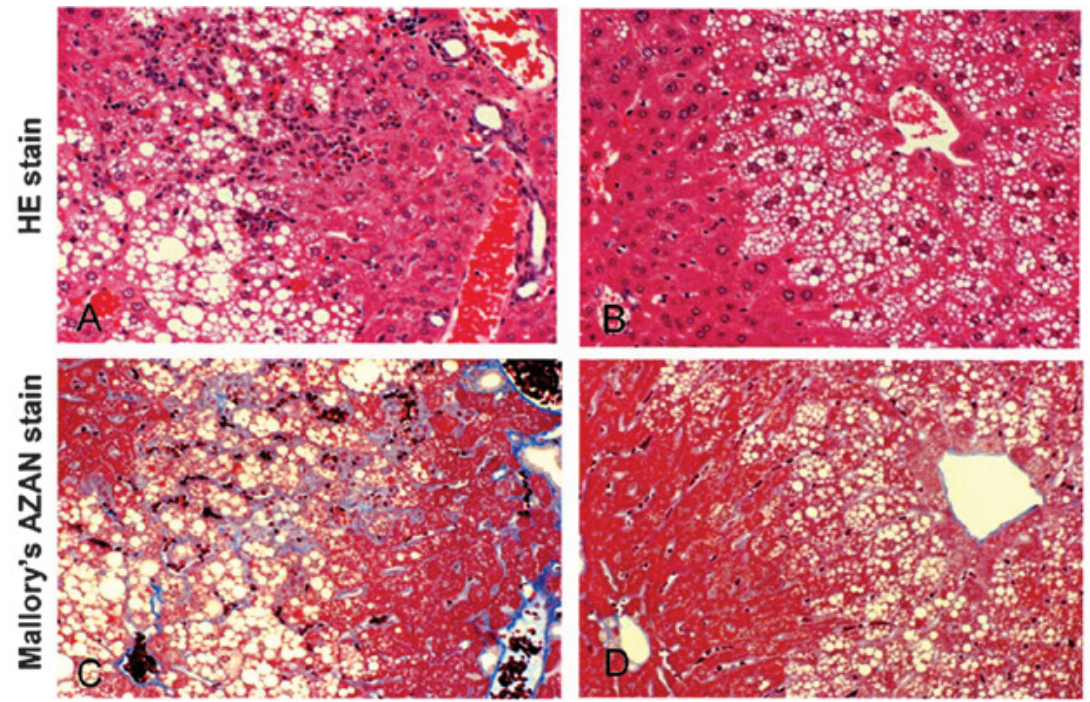

$\mathrm{X} 100$

nSREBP-1c Tg

nSREBP-1c/adiponectin Tg

Figure 4. Light microscopy findings. (A and B) Fat droplets, cell infiltration and pericellular fibrosis were decreased in the nSREBP-1c/adiponectin transgenic mice compared with the (C and D) nSREBP-1c transgenic mice.
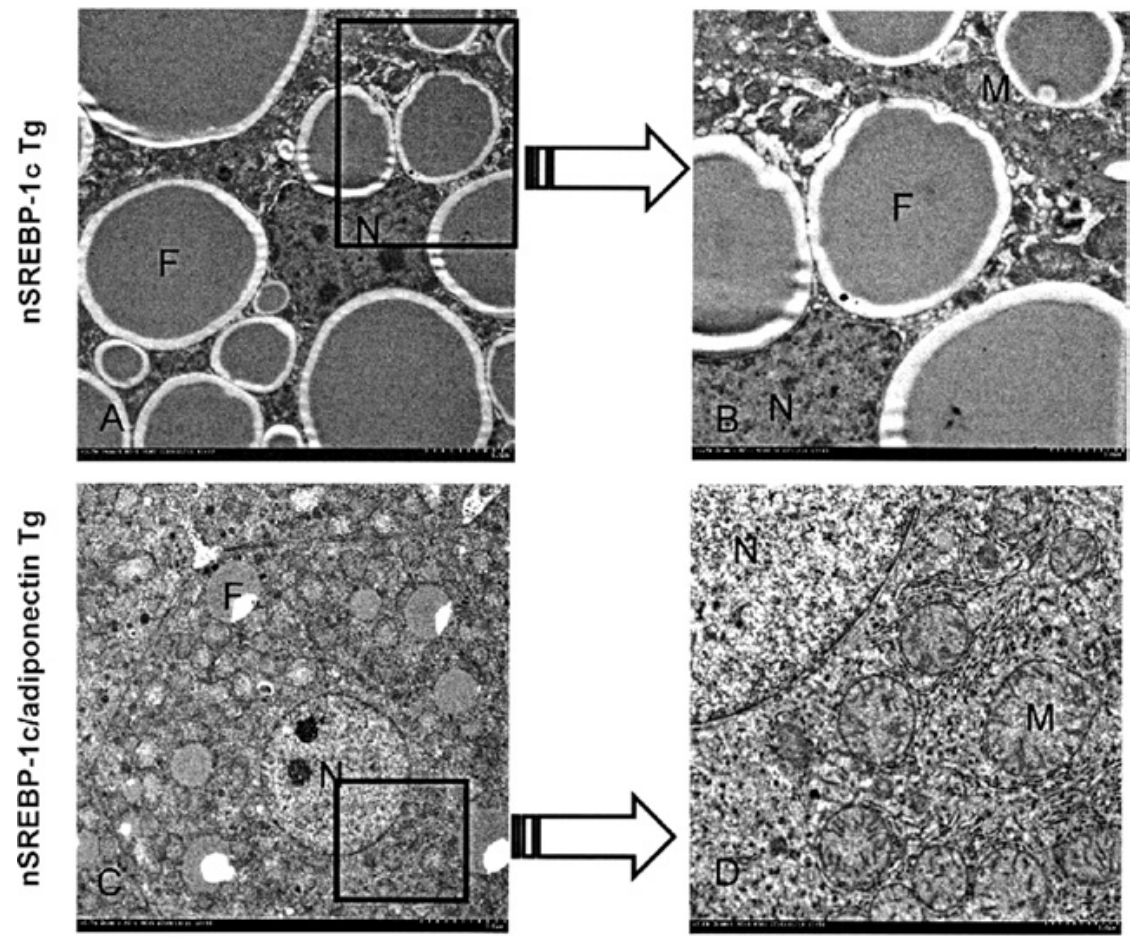

Figure 5. Electron microscopy findings. (A and B) In the liver of the SREBP-1c transgenic mice, hepatocytes with degenerated nuclei, small and large fat droplets, and, in the magnified image, enlarged endoplasmic reticula are visible. (C and D) However, in the liver of the nSREBP-1c and adiponectin doubletransgenic mouse, only a few small fat droplets are located in the cytoplasm of the hepatocytes. However, the nuclei and organelles, such as mitochondria and ER, are similar to those of normal hepatocytes.

nSREBP-1c transgenic mice. However, ATF-6 protein expression in the three groups was almost the same (Fig. 7B).

\section{Discussion}

Our study shows that ER stress is enhanced in the liver of nSREBP-1c transgenic NASH model mice. Our findings also indicate that adiponectin suppresses ER stress through the XBP-1, ATF-4 and ACC pathway in the liver of the nSREBP- 1c/adiponectin transgenic mice. Adiponectin is known to suppress ER stress in obesity and diabetes $(16,17)$. Recent studies have linked ER stress to obesity, insulin action and type 2 diabetes $(18,19)$, and the activation of the UPR is governed by three ER transmembrane proteins: inositol-requiring kinase-1 (IRE1), protein kinase-like ER kinase (PERK) and activating transcription factor-6 (ATF6). Upon ER stress, IRE1 endonuclease facilitates the splicing of XBP-1 mRNA, leading to the translation of an activated version of the transcription 
A

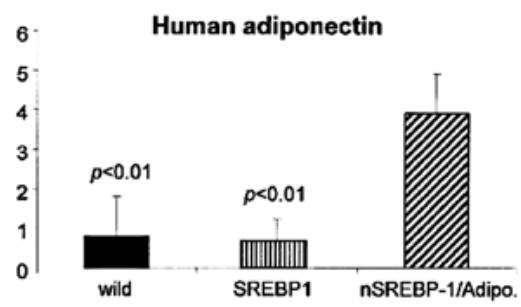

C

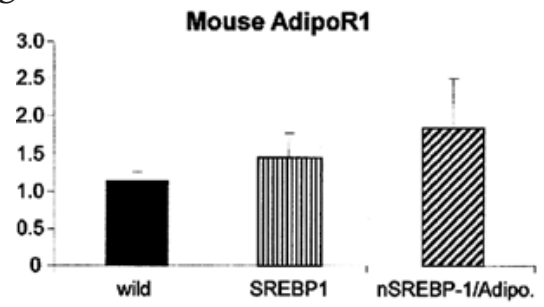

B

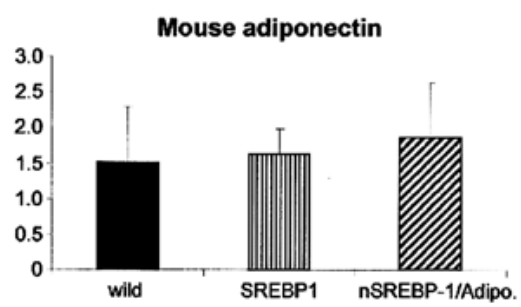

D

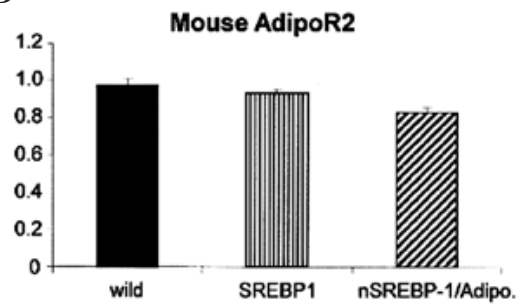

Figure 6. Real-time PCR analysis. (A) Human adiponectin expression was significantly increased in the liver of nSREBP-1c/adiponectin transgenic mice compared with the wild-type and nSREBP-1c transgenic mice. (B) However, there was no significant difference in the mouse adiponectin mRNA expression between the wild type, nSREBP-1c transgenic mice, and nSREBP-1c/adiponectin transgenic mice. (C and D) There was no significant difference between the nSREBP-1c transgenic mice and nSREBP-1c/adiponectin transgenic mice in mouse AdipoR1 and 2 expression levels.

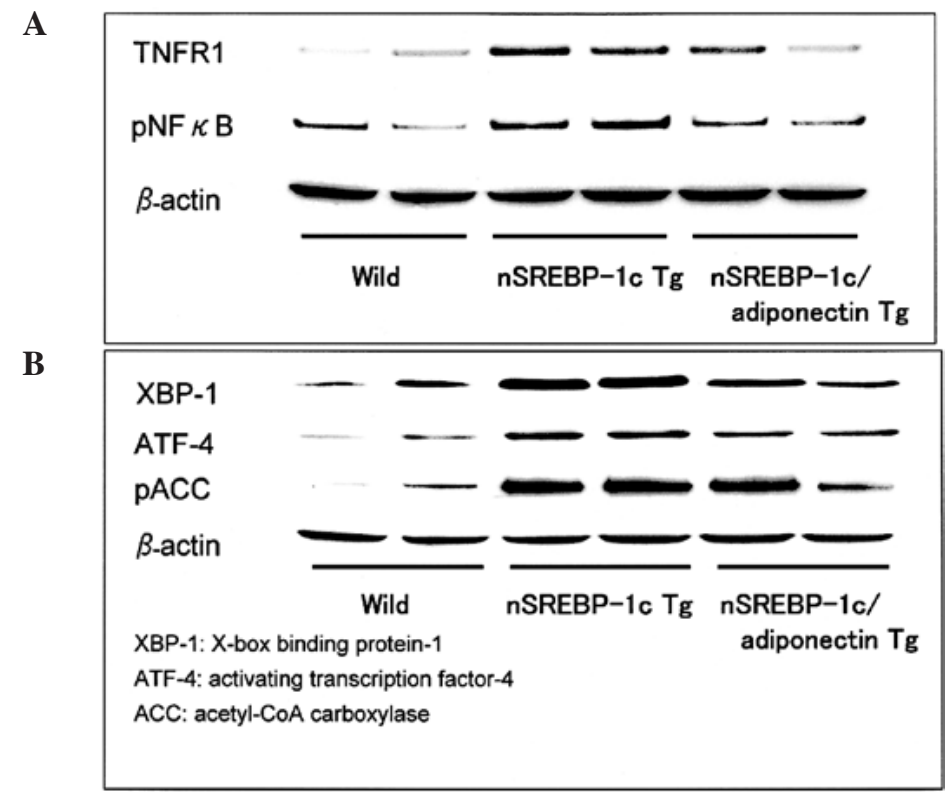

Figure 7. Western blot analysis. TNFR1 and phosphorylated NFKB were decreased in the liver of the nSREBP-1c/adiponectin transgenic mice compared with the nSREBP-1c transgenic mice. (A) It can be assumed that adiponectin suppresses the inflammation through the TNFR1 and NFkB pathway in the liver of the nSREBP-1c/adiponectin transgenic mice. In addition, the protein expression of XBP-1, ATF-4 and phosphorylated ACC were decreased in the liver of the nSREBP-1c/adiponectin transgenic mice compared with nSREBP-1c transgenic mice. (B) However, ATF-6 protein expression in the three groups was almost the same.

factor XBP-1. XBP-1's binding of one of the PI3K regulatory subunits, p85 $\alpha$ or p $85 \beta$, enhances nuclear entry of this transcription factor and induction of UPR target genes $(20,21)$. The p85 subunit does not directly modulate other arms of the UPR, including the association of IRE1 with TRAF2 and the subsequent activation of JNK and PERK phosphorylation of eIF2 (p-eIF2), which represses protein synthesis coincident with preferential translation of the UPR transcriptional activator ATF4. However, the ATF6 arm of the UPR, which is involved in proteolytic cleavage and release of the transcription factor into the nucleus, may be activated through association with p85 (22).

Although hypoadiponectinemia has been implicated in the development of human nonalcoholic fatty liver disease (NAFLD) and NASH (23), there was no direct evidence that adiponectin has preventive effects on spontaneously occurring NASH. In this study, the double-transgenic mice producing adiponectin in the liver showed normal or slightly elevated 
serum levels of adiponectin. The double-transgenic mice normally expressed the two subtypes of AdipoRs, that is, AdipoR1 and 2, in the liver. Adiponectin expressed in the liver stimulates adenosine monophosphate-activated protein kinase activation and PPAR $\alpha$ signaling pathways through AdipoR1 and 2 , respectively.

Adiponectin may also exert a protective effect through its anti-inflammatory action (9). Adiponectin is an adipocytokine secreted by mature adipocytes, and its receptors are widely distributed in a number of tissues, including the liver. Adiponectin has direct actions in the liver with prominent roles in improving hepatic insulin sensitivity, increasing fatty acid oxidation and decreasing inflammation. Adiponectin-null mice exhibit impaired liver regeneration and increased hepatic steatosis (24). Yoshiuchi et al directly evaluated the effects of the diabetic agent pioglitazone on in vivo ER stress under diabetic conditions. Pioglitazone treatment reduced the accumulation of fat droplets in the liver and attenuated the development of insulin resistance, that is, pioglitazone suppresses ER stress in the liver (25). Consequently, adiponectin may directly improve the pathogenesis of NAFLD, including NASH.

In conclusion, the transgenic mouse expressing nSREBP-1c in adipose tissue may serve as a unique model of NASH. The transgene of adiponectin in the liver of the nSREBP-1c transgenic mice induced the improvement of insulin resistance, ER stress, and intralobular inflammation and fibrosis. These observations may lead to the development of novel therapies for NASH in humans.

\section{References}

1. Lee AH and Glimcher LH: Intersection of the unfolded protein response and hepatic lipid metabolism. Cell Mol Life Sci 66: 2835-2850, 2009.

2. Basseri S and Austin RC: ER stress and lipogenesis: a slippery slope toward hepatic steatosis. Dev Cell 15: 795-796, 2008.

3. Rutkowski DT, Wu J, Back SH, Callaghan MU, Ferris SP, Iqbal J, Clark R, Miao H, Hassler JR, Fornek J, Katze MG, Hussain MM, Song B, Swathirajan J, Wang J, Yau GD and Kaufman RJ: UPR pathways combine to prevent hepatic steatosis caused by ER stress-mediated suppression of transcriptional master regulators. Dev Cell 15: 829-840, 2008

4. Ron D and Walter P: Signal integration in the endoplasmic reticulum unfolded protein response. Nat Rev Mol Cell Biol 8: 519-529, 2007

5. Ji C: Dissection of endoplasmic reticulum stress signaling in alcoholic and non-alcoholic liver injury. J Gastroenterol Hepatol 23: S16-S24, 2008.

6. Colgan SM, Tang D, Werstuck GH and Austin RC: Endoplasmic reticulum stress causes the activation of sterol regulatory element binding protein-2. Int J Biochem Cell Biol 39: 1843-1851, 2007.

7. Goldstein JL, DeBose-Boyd RA and Brown MS: Protein sensors for membrane sterols. Cell 124: 35-46, 2006.

8. Nakayama H, Otabe S, Ueno T, Hirota N, Yuan X, Fukutani T, Hashinaga T, Wada $\mathrm{N}$ and Yamada $\mathrm{K}$ : Transgenic mice expressing nuclear sterol regulatory element-binding protein $1 \mathrm{c}$ in adipose tissue exhibit liver histology similar to nonalcoholic steatohepatitis. Metabolism 56: 470-475, 2007.

9. Mendez-Sanchez N, Chavez-Tapia NC, Zamora-Valdes and Uribe M: Adiponectin, structure, function and pathophysiological implications in non-alcoholic fatty liver disease. Mini Rev Med Chem 6: 651-656, 2006.
10. Yamauchi T, Kamon J, Waki H, Terauchi Y, Kubota N, Hara K, Mori Y, Ide T, Murakami K, Tsuboyama-Kasaoka N, et al: The fat-derived hormone adiponectin reverses insulin resistance associated with both lipoatrophy and obesity. Nat Med 7: 941-946, 2001

11. Nakayama H, Otabe S, Yuan X, Ueno T, Hirota N, Fukutano T, Wada N, Hashinaga T and Yamada K: Effects of adiponectin transgenic expression in liver of nonalcoholic steatohepatitis model mice. Metabolism 58: 901-908, 2009.

12. Awazawa M, Ueki K, Inabe K, Yamauchi T, Kaneko K, Okazaki Y, Bardeesy N, Ohnishi S, Nagai R and Kadowaki T: Adiponectin suppresses hepatic SREBP1c expression in an AdipoR1/LKB1/AMPK dependent pathway. Biochem Biophys Res Commun 382: 51-56, 2009.

13. Shimomura I, Hammer RE, Richardson JA, Ikemoto S, Bashmakov Y, Goldstein JH, Goldstein JL and Brown MS: Insulin resistance and diabetes mellitus in transgenic mice expressing nuclear SREBP-1c in adipose tissue: model for congenital generalized lipodystrophy. Genes Dev 12: 3182-3194, 1998.

14. Otabe S, Yuan X, Fukutani T, Wada N, Hashinaga T, Nakayama H, Hirota N, Kojima M and Yamada K: Overexpression of human adiponectin in transgenic mice results in suppression of fat accumulation and prevention of premature death by high-calorie diet. Am J Physiol Endocrinol Metab 293: E210-E218, 2007.

15. Matteoni CA, Younossi ZM, Gramlich T, Boparai N, Liu YC and McCullough AJ: Nonalcoholic fatty liver disease: a spectrum of clinical and pathological severity. Gastroenterology 116: 1413-1419, 1999.

16. Hosogai N, Fukuhara A, Oshima K, Miyata Y, Tanaka S, Segawa K, Furukawa S, Tochino Y, Komuro R, Matsuda M and Shimomura I: Adipose tissue hypoxia in obesity and its impact on adipocy tokine dysregulation. Diabetes 56: 901-911, 2007.

17. Frizzell N, Lima M and Baynes JW: Succination of proteins in diabetes. Free Radic Res 45: 101-109, 2011.

18. Ozcan U, Cao Q, Yilman E, Lee AH, Iwakoshi NN, Ozdelen E, Görgün C, Glimcher LH and Hotamisligil GS: Endoplasmic reticulum stress links obesity, insulin action, and type 2 diabetes. Science 306: 457-461, 2004.

19. Wek RC and Anthony TG: Obesity: stressing about unfolded proteins. Nat Med 16: 374-376, 2010

20. Park SW, Zhou Y, Lee J, Lu A, Sun C, Chung J, Ueki K and Ozcan U: The regulatory subunits of PI3K, p85 $\alpha$ and $\mathrm{p} 85 \beta$, interact with XBP-1 and increase its nuclear translocation. Nat Med 16: 429-437, 2010.

21. Winnay JH, Boucher J, Mori MA, Ueki K and Kahn R: A regulatory subunit of phosphoinositide 3-kinase increases the nuclear accumulation of X-box-binding protein-1 to moderate the unfolded protein response. Nat Med 16: 438-445, 2010.

22. Van Herpen NA and Schrauwen-Hinderling VB: Lipid accumulation in non-adipose tissue and lipotoxicity. Physiol Behav 94: 231-241, 2008.

23. Bugianesi E, Pagotto U, Manini R, Vanni E, Gastaldelli A, de Lasio R, Pasquali R, Cassio A, Cicognani A and Cacciari E. Plasma adiponectin in nonalcoholic fatty liver is related to hepatic insulin resistance and hepatic fat content, not to liver disease severity. J Clin Endocrinol Metab 90: 3498-3504, 2005.

24. Shu RZ, Zhang F, Wang F, Feng DC, Li XH, Ren WH, Wu XL, Yang X, Liao XD, Huang L and Wang ZG: Adiponectin deficiency impairs liver regeneration through attenuating STAT3 phosphorylation in mice. Lab Invset 89: 1043-1052, 2009.

25. Yoshiuchi K, Kaneko H, Matsuoka T, Kasami R, Kohono K, Iwawaki T, Nakatani Y, Yamasaki Y, Shimomura I and Matsuhisa M: Pioglitazone reduces ER stress in the liver: direct monitoring of in vivo ER stress using ER stress-activated indicator transgenic mice. Endocr J 56: 1103-1111, 2009. 